Pacific Journal of Mathematics

ON THE HOLOMORPHY OF MAPS FROM A COMPLEX TO A 


\title{
ON THE HOLOMORPHY OF MAPS FROM A COMPLEX TO A REAL MANIFOLD
}

\author{
SUBHASHIS NAG
}

Let $f: X \rightarrow Y$ be a $C^{1}$-submersion from a complex manifold $X$ to a real $C^{1}$-manifold $Y$. One main object of this paper is to prove two sets of necessary and sufficient conditions which will guarantee that $Y$ can be equipped with a complex structure making $f$ holomorphic. We provide a generic counterexample to show the essential nature of the conditions we establish.

The second set of conditions (Theorem 3) apply even when $X$ is a complex Banach manifold. This theorem is then used to prove the existence of the natural complex structure on the Teichmuller spaces of Riemann surfaces of finite type.

We shall start by giving some necessary conditions in Lemmas 1, 2, 3 .

LEMMA 1. If $f: X^{m} \rightarrow Y^{d}$ is a surjective holomorphic submersion between complex manifolds of dimensions $m$ and $d$ respectively then the fibers of $f$ (i.e. the sets $f^{-1}(y), y \in Y$ ), are (closed) complex submanifolds in $X$ of dimension $(m-d)$.

Proof. This is immediate from the implicit function theorem in the holomorphic category.

Let $X^{m}$ be an $m$-dimensional complex manifold. We let $\mathrm{Gr}_{(m-d)}(T X)$ denote the Grassmann bundle of $(m-d)$-dimensional complex subspaces in the tangent bundle $T X$ of $X$. The total space $\operatorname{Gr}_{(m-d)}(T X)$ inherits a natural complex structure from $X$.

Definition 1. An $(m-d)$-dimensional distribution on $X$ is a section of the $\mathrm{Gr}_{(m-d)}(T X)$ bundle over $X$. We say the distribution is analytic if the section is an analytic function.

REMARK. Note that the distribution is analytic if and only if it can be spanned locally by $(m-d)$ linearly independent analytic vector fields.

Let $f: X \rightarrow Y$ be a $C^{1}$-submersion from a complex manifold $X$ onto a real $C^{1}$-manifold $Y$. Then if $y \in Y$ and $x \in f^{-1}(y)$, the differential of $f$ at $x d_{x} f: T_{x} X \rightarrow T_{y} Y$ is a surjective linear map. If ker $d_{x} f$ is a complex 
subspace of $T_{x} X$ then (and only then) does $T_{y} Y$ inherit a complex vector-space structure such that $d_{x} f$ is C-linear. (Indeed, $d_{x} f$ : $T_{x} X / \operatorname{ker} d_{x} f \rightarrow T_{y} Y$ is then a C-linear isomorphism.) This leads to the following definition:

DEFINITION 2. If for all $y \in Y T_{y} Y$ inherits via $d_{x} f$ a unique complex structure independent of the choice of $x \in f^{-1}(y)$, then we say that $f$ induces a well-defined almost complex structure on $Y$. In this definition we allow $X$ and $Y$ to be complex Banach manifolds.

Definition 3. Let $Y$ be a $C^{1}$-manifold. A complex structure $\sigma$ on $Y$ will be said to be compatible with the $C^{1}$-structure if the $C^{1}$-structure underlying $\sigma$ coincides with the original $C^{1}$-structure on $Y$.

LEMMA 2. If $f: X^{m} \rightarrow Y^{d}$ is a surjective holomorphic submersion between complex manifolds of dimensions $m$ and $d$, then the distribution $\Delta$ on $X, \Delta$ : $X \rightarrow \mathrm{Gr}_{(m-d)}(T x)$ given by

$$
\begin{aligned}
\Delta(x) & =\operatorname{ker} d_{x} f \\
& =\text { tangent space at } x \text { to the fiber } f^{-1}(f(x))
\end{aligned}
$$

is an analytic distribution.

Proof. By Lemma 1, $\Delta$ defines a distribution. We will show $\Delta$ is analytic by a computation.

In terms of local coordinates $\left(\xi^{1}, \ldots, \xi^{m}\right)$ and $\left(\eta_{1}, \ldots, \eta_{d}\right)$ (around $x_{0}$ and $f\left(x_{0}\right)$ respectively) let $f$ be represented by $d$ holomorphic functions

$$
\eta_{j}=f_{J}\left(\xi^{1}, \ldots, \xi^{m}\right), \quad j=1,2, \ldots, d .
$$

Then for $x$ near $x_{0}$ we have,

$$
\begin{aligned}
\Delta(x) & =\operatorname{ker} d_{x} f=\left\{\alpha^{i} \frac{\partial}{\partial \xi^{i}}: d f\left(\alpha^{l} \frac{\partial}{\partial \xi^{l}}\right)=0\right\} \\
& =\left\{\alpha^{i} \frac{\partial}{\partial \xi^{i}}: \alpha^{i}\left(\frac{\partial f_{j}}{\partial \xi^{l}}\right) \frac{\partial}{\partial \eta_{j}}=0, \text { for all } j\right\}
\end{aligned}
$$

(summation convention in use). 
The $d$ homogeneous linear equations

$$
\sum_{i=1}^{m} \alpha^{2} \frac{\partial f_{j}}{\partial \xi^{i}}=0, \quad j=1, \ldots, d,
$$

in the $m$ unknowns $\alpha_{i}$ have their coefficient matrix $\left(\partial f_{j} / \partial \xi^{l}\right)$ holomorphically dependent on $x$ and of maximal rank $d$ (as $f$ is submersive). Hence the solution space is spanned by $(m-d)$ linearly independent solutions which depend holomorphically on $x$ in a neighbourhood of $x_{0}$. The result follows.

LEMMA 3. Let $f: X \rightarrow Y$ be a surjective $C^{1}$-submersion from a complex Banach manifold $X$ to a $C^{1}$-manifold $Y$. If $Y$ has a complex structure $\sigma$ compatible with its $C^{1}$-structure making $f$ holomorphic, then $\sigma$ is the unique such complex structure and $f$ induces a well-defined almost complex structure on $Y$ (this almost complex structure being of course the one underlying $\sigma$ ).

The proof of this follows directly from Definition 3.

When $f$ has connected fibers the necessary conditions of Lemmas 1 and 2 become sufficient to guarantee a complex structure on $Y$ making $f$ a holomorphic submersion.

THEOREM 1. Let $f: X \rightarrow Y$ be a surjective $C^{1}$-submersion from an m-dimensional complex manifold $X$ to a 2 d-dimensional $C^{1}$-manifold $Y$. Suppose

(1) The fiber $f^{-1}(y)$, for each $y \in Y$, is a $(m-d)$-dimensional connected complex submanifold of $X$;

(2) the $(m-d)$-dimensional distribution on $X$ given by $\Delta(x)=$ tangent space to the fiber $f^{-1}(f(x))$ at $x$ is an analytic distribution.

Then $Y$ has a unique complex structure with respect to which $f$ is holomorphic. This structure is compatible with the $C^{1}$-structure and $f$ becomes a holomorphic submersion.

Proof. The $(m-d)$-dimensional fibers are precisely the integrating manifolds for the distribution $\Delta$. So $\Delta$ is an involutive analytic distribution. Invoking the Frobenius theorem (in the holomorphic category) we know that for any $x_{0} \in X$ there is a 'cubic coordinate system' in a neighbourhood $U$ of $x_{0}$, with coordinate function $z=\left(z_{1}, \ldots, z_{m}\right)$ such that the slices

$$
F_{c_{1}, \ldots, c_{d}}=\left\{\left(z_{1}, \ldots, z_{m}\right) \in z(U): z_{1}=c_{1}, z_{2}=c_{2}, \ldots, z_{d}=c_{d}\right\}
$$


(for values of $\left(c_{1}, \ldots, c_{d}\right)$ in the open subset of $\mathbf{C}^{d}$ spanned by the first $d$ coordinates in $U$ ), are integral manifolds of $\Delta$. (Note: The $C^{\infty}$ version of the above theorem is Theorem 1.60 of Warner [5]; the analytic version has the same proof in the analytic category. Also recall that a cubic coordinate system $(U, z)$ centered at $x_{0}$ is a coordinate system such that $z(U)$ is an open cube around the origin in $\mathbf{C}^{m}$ and $z\left(x_{0}\right)=$ origin.)

Now, the integrating manifolds for $\Delta$ in $U$ are the fibers $f^{-1}(y) \cap U$ for $y \in f(U)$. So to each $y \in f(U)$ we can associate uniquely the $\left(c_{1}, \ldots, c_{d}\right)$ such that

$$
z\left(f^{-1}(y) \cap U\right)=F_{\left(c_{1}, \ldots, c_{d}\right)} .
$$

The map $\varphi,\left(c_{1}, \ldots, c_{d}\right) \stackrel{\varphi}{\mapsto}$ (corresponding $y$ ) from the open set in $\mathbf{C}^{d} \rightarrow f(U)$ is easily seen to be continuous and injective. By invariance of domain then, (see Dold [1]), $\varphi$ is an open map onto an open set in $Y$. So $f(U)$ is open in $Y$. Thus, via $\varphi$ we have given a complex coordinate system on the open set $f(U)$ in $Y$.

Claim. This method of assignment of complex coordinates on $Y$ gives it a structure of a complex manifold compatible with its $C^{1}$-structure.

Indeed suppose $(U, z)$ and $(V, w)$ are two cubic coordinate systems in $X$ obtained from the Frobenius theorem as above.

On $U \cap V$ (assumed non-empty) the first $d$ coordinates of $z$, $\left(z_{1}, \ldots, z_{d}\right)$ are biholomorphically related to the first $d$ coordinates of $w$, $\left(w_{1}, \ldots, w_{d}\right)$. This is because $\left(z_{1}, \ldots, z_{m}\right)$ and $\left(w_{1}, \ldots, w_{m}\right)$ are biholomorphically related and the slices from each are the fibers of $f$ by construction. This easily implies that the first $d$ coordinates in each are related to the first $d$ in the other.

Now suppose a neighbourhood of $y_{0} \in Y$ has coordinates assigned from two Frobenius cubic coordinate systems $U$ and $V$ in $X$. Say $f\left(x_{0}\right)=$ $f\left(x_{0}^{\prime}\right)=y_{0}, x_{0} \in U, x_{0}^{\prime} \in V$. Even if $U \cap V=\varnothing$ we can choose a finite sequence of Frobenius cubic coordinate systems $U=U_{0}, U_{1}, \ldots, U_{n}=V$ such that

$$
U_{j} \cap U_{j+1} \neq \varnothing \text {. }
$$

This follows from the connectedness of $f^{-1}\left(y_{0}\right)$; indeed we can choose a compact path joining $x_{0}$ to $x_{0}^{\prime}$ on $f^{-1}\left(y_{0}\right)$, and each point on this path has Frobenius cubic coordinate system around it. By a standard compactness argument we are done. Hence, since the first $d$ coordinate of $U_{j}$ are holomorphically related to the first $d$ coordinate of $U_{j+1}$, for all $j$ (by our previous argument), we see that the coordinates around $y_{0}$ obtained from 
$U$ and $V$ are holomorphically related. This shows that the complex structure on $Y$ is well-defined. The compatibility with the $C^{1}$-structure is trivial.

Now, in terms of the Frobenius cubic coordinates $\left(z_{1}, \ldots, z_{m}\right)$ in $U \subset X$ and the induced chart on $f(U) \subset Y, f$ simply becomes projection to the first $d$ coordinates. Hence $f$ is a holomorphic submersion with respect to this complex structure on $Y$.

Uniqueness. Call the complex structure obtained above $\sigma$, and suppose there is another complex structure $\sigma^{\prime}$ making $f$ holomorphic. If we prove that the identity map 1: $Y_{\sigma} \rightarrow Y_{\sigma^{\prime}}$ is holomorphic we will have shown $\sigma=\sigma^{\prime}$ because 1 is a homeomorphism, and any holomorphic homeomorphism is biholomorphic, (see Herve [3, pg. 109, Corollary 2]).

To prove $1: Y_{\sigma} \rightarrow Y_{\sigma^{\prime}}$ is holomorphic, consider $(U, z)$ a Frobenius cubic coordinate system in $X$. If we fix the last $(m-d)$ coordinates in $z$ at some values, $z_{d+1}=k_{d+1}, z_{d+2}=k_{d+2}, \ldots, z_{m}=k_{m}$, then we obtain a $d$-dimensional slice, say $S$, of $U$ which is a $d$-dimensional complex submanifold in $U$ projecting holomorphically and homeomorphically under $f$ to the open set $f(U)$ in $Y_{\sigma}$. Hence $\left.f\right|_{S}$ has a holomorphic inverse $g$ from $f(U) \subset Y_{\sigma}$ to $S$ again by the theorem from Herve [3] used above. But $S$ is a complex submanifold in $X$; so, since $f: X \rightarrow Y_{\sigma^{\prime}}$ is assumed holomorphic we see $\left.f\right|_{S}: S \rightarrow Y_{\sigma^{\prime}}$ is also holomorphic. Thus $\left.f\right|_{S} \circ g=$ $\left.1\right|_{f(U)}: f(U)_{\sigma} \rightarrow f(U)_{\sigma^{\prime}}$ is holomorphic. This completes the proof.

We have now completely proved the following.

THEOREM 2. Let $f: X \rightarrow Y$ be a surjective $C^{1}$-submersion from an m-dimensional complex manifold to a 2 d-dimensional $C^{1}$-manifold with fibers $f^{-1}(y)$ connected for all $y \in Y$. Then there is a complex structure on $Y$, compatible with the $C^{1}$-structure, making $f$ holomorphic, if and only if the following conditions hold:

(1) The fibers are $(m-d)$-dimensional complex submanifolds in $X$ and

(2) the distribution on $X$ given by $\Delta(x)=$ tangent space to the fiber through $x$ is analytic.

The complex-structure, when it exists, is unique. Another set of necessary sufficient conditions are below.

THEOREM 3. Let $f: X \rightarrow Y$ be a surjective $C^{1}$-submersion from a complex Banach manifold $X$ (modelled on a complex Banach space B) to a 
$2 d$-dimensional $C^{1}$-manifold $Y$. Then there is a complex structure on $Y$, compatible with its $C^{1}$-structure, making $f$ holomorphic if and only if $f$ induces a well-defined almost complex structure on $Y$.

\section{The complex structure, when it exists, is unique.}

Proof. In view of Lemma 3 we only need to prove sufficiency of the condition. At any $x \in X$ look at ker $d_{x} f$ which is a complex subspace of $T_{x} X$ (note the discussion preceding Definition 2). Here $T_{x} X$ can be identified as $B$. By linear algebra there exists a $d$-dimensional complex subspace, say $G_{x}$, of $B$ which is complementary to $\operatorname{ker} d_{x} f$.

Via local coordinates around $x \in X$ we can identify a neighbourhood of $x$ with a neighbourhood in $B$. Then, under this identification, we can consider an open subset $\Gamma_{x}$ of $G_{x}$ as sitting in $X$ and containing $x$. We may then consider the restriction of $f$ to $\Gamma_{x}$. Since $G_{x}$ is finite dimensional it is closed in $B$, hence $\left.f\right|_{\Gamma_{x}}$ is $C^{1}$ on $\Gamma_{x}$. By the inverse function theorem there exists an open neighbourhood $U_{x}$ of $x$ in $\Gamma_{x}$ so that $\left.f\right|_{U_{x}}$ is a $C^{1}$-diffeomorphism from $U_{x}$ to the open set $f\left(U_{x}\right)$ in $Y$. Thus $\left.f\right|_{U_{x}}$ assigns complex coordinates to this open neighbourhood $f\left(U_{x}\right)$ of $f(x) \in Y$ since $U_{x}\left(\subset G_{x} \cong \mathbf{C}^{d}\right)$ can be identified as an open subset of $\mathbf{C}^{d}$.

We claim that this method of assignment of complex coordinates to $Y$ makes $Y$ a complex manifold. For suppose two neighbourhoods of $y \in Y$ have complex coordinates coming from $U_{x}$ and $U_{x^{\prime}}$ as explained above with $f(x)=f\left(x^{\prime}\right)=y$. The coordinate transition $\left.\left(\left.f\right|_{U_{x}}\right)^{-1} \circ f\right|_{U_{x}}$ from $U_{x}$ to $U_{x^{\prime}}$ is $\xi \mapsto \xi^{\prime}$ where $f(\xi)=f\left(\xi^{\prime}\right)$. This is a $C^{1}$-map and its derivative $\left(\left.d_{\xi^{\prime}} f\right|_{U_{x}}\right)^{-1} \circ\left(\left.d_{\xi} f\right|_{U_{x}}\right)$ is $\mathbf{C}$-linear because we assumed that $f$ induces a well-defined almost complex structure on $Y$. So the transition function is proved holomorphic, (being a $C^{1}$-map with a $\mathbf{C}$-linear derivative), as required.

Finally, $f$ looked at in one of these coordinate charts obtained above simply becomes a restriction of the C-linear projection map $\pi_{2}: B \rightarrow G_{x}$ (in the direct sum decomposition $\left.B=\operatorname{ker} d_{x} f \oplus G_{x}\right)$. Now since $G_{x}$ is finite dimensional and the null-space of $\pi_{2}\left(=\operatorname{ker} d_{x} f\right)$ is closed in $B$ it follows from topological vector space theory that $\pi_{2}$ is continuous. Since $f$ is identified as a restriction of the linear map $\pi_{2}$ the derivative of $f$ is also identifiable as $\pi_{2}$. So $d f$ exists and is a continuous C-linear map, proving that $f$ is a holomorphic submersion in these charts.

Uniqueness of the complex-structure. An argument exactly as in the uniqueness proof for Theorem 1 works here also. 
REMARK. It turns out that a map which can be made holomorphic as in the situations of Theorems 1 and 3 has peculiar rigidity properties. Indeed if $f$ satisfies the hypothesis of Theorem 1 (or the condition of Theorem 4) then the $C^{1}$-structure assigned to $Y$ is the unique $C^{1}$-structure making $f$ a $C^{1}$-submersion. This is because the $C^{1}$-structure is the one underlying the unique complex structure that makes $f$ holomorphic. Essentially this reflects the fact that the map $f$ is in our situation a diffeomorphism between a transverse to its fibers and an open set in $Y$.

An application of Theorem 3 to the existence of the natural complex structure on Teichmuller spaces. The Teichmuller spaces are moduli spaces for Riemann surfaces of a fixed type.

Let $U$ be the upper half-plane and $\Gamma$ a fixed-point free Fuchsian group of first kind acting on $U$ so that $S_{0}=U / \Gamma$ is a Riemann surface of type $(g, k)$ (i.e. genus $g$ with $k$ punctures). The group $\Gamma$ is our 'base point' in Teichmuller space. We provide below the definition of Teichmuller space for type $(g, k)$ surfaces, $2 g-2+k>0$ (see also Earle [2]). Let

$$
L^{\infty}(\Gamma)=\left\{\mu \in L^{\infty}(U): \mu(\gamma z) \overline{\gamma^{\prime}(z)} / \gamma^{\prime}(z)=\mu(z) \text { a.e. for all } \gamma \in \Gamma\right\}
$$

and set $M(\Gamma)=$ open unit ball in the complex Banach space $L^{\infty}(\Gamma)$ (these are the 'Beltrami differentials' for the group $\Gamma)$. Let $w_{\mu}(\mu \in M(\Gamma))$ be the unique quasiconformal (q.c.) homeomorphism of $U$ solving the Beltrami equation $w_{\bar{z}}=\mu w_{z}$ and normalized by the requirement of fixing $0,1, \infty$. We say $\mu$ is the complex dilatation of $w$. Now the maps $w_{\mu} \circ \gamma \circ w_{\mu}^{-1}=\gamma_{\mu}$ form again a Fuchsian group $\Gamma_{\mu}$ as $\gamma$ ranges through $\Gamma$, and $U / \Gamma_{\mu}={ }^{\prime} S_{\mu}$ ' is a Riemann surface of type $(g, k)$ also. We define an equivalence relation $\sim$ in $M(\Gamma)$ as follows: $\mu \sim \nu$ for $\mu$ and $\nu \in M(\Gamma)$ if $\gamma_{\mu}=\gamma_{\nu}$ for all $\gamma \in \Gamma$; now we set the Teichmuller space $T(\Gamma)=M(\Gamma) / \sim$. Notice that $w_{\mu}$ induces a 'marking map' $f_{\mu}: S_{0} \rightarrow S_{\mu}$. The idea of the relation $\sim$ is that $\mu \sim \nu$ if and only if $S_{\mu}$ is conformally equivalent to $S_{\nu}$ by a conformal mapping homotopic to $f_{\nu} \circ f_{\mu}^{-1}$. This shows why $T(\Gamma)$ as defined is a moduli space for Riemann surfaces.

Consider the quotient map $\Phi: M(\Gamma) \rightarrow T(\Gamma)$. It is a classical question (with an affirmative answer) whether $T(\Gamma)$ carries a complex structure making $\Phi$ a holomorphic submersion. We will use Theorem 3 to show that there is indeed such a (unique) complex structure compatible with the $C^{1}$-structure that $T(\Gamma)$ inherits from $M(\Gamma)$. In the process we find an interesting representation of the tangent space to the fibers of $\Phi$ at any point in $M(\Gamma)$.

These $T(\Gamma)$ are known to be finite dimensional (real dimension $=6 g$ $-6+2 k$ by Teichmuller's theorem) and the subspace ker $d_{0} \Phi \subset L^{\infty}(\Gamma)$ 
was known (as given below) in 'Teichmuller's lemma' without any appeal to existence of a complex structure on $T(\Gamma)$. Indeed,

$$
\operatorname{ker} d_{0} \Phi=\left\{\mu \in L^{\infty}(\Gamma): \iint_{U / \Gamma} \mu \varphi=0, \text { for all } \varphi \in Q(\Gamma)\right\}
$$

where

$$
\begin{aligned}
Q(\Gamma)= & \text { integrable holomorphic quadratic differentials for } \Gamma \\
= & \text { holomorphic functions } \varphi \text { on } U: \varphi(\gamma z) \gamma^{\prime}(z)^{2}=\varphi(z) \\
& \text { for all } z \in U, \text { and } \gamma \in \Gamma\} .
\end{aligned}
$$

So $K_{0}=\operatorname{ker} d_{0} \Phi$ is seen to be a complex subspace of $L^{\infty}(\Gamma)$. Now let $\theta \in M(\Gamma)$ be any other point on the fiber of $\Phi$ above $\Phi(0)$. We wish to compare $\operatorname{ker} d_{\theta} \Phi$ with $\operatorname{ker} d_{0} \Phi$ to understand whether $\Phi$ induces a well-defined almost complex structure on $T(\Gamma)$.

Now, if we take the group $\Gamma_{\theta}=w_{\theta} \circ \Gamma \circ w_{\theta}^{-1}$ as our base point for defining Teichmuller space then we get a corresponding quotient map $\Phi_{\theta}$ : $M\left(\Gamma_{\theta}\right) \rightarrow T\left(\Gamma_{\theta}\right)$, and of course

$$
K_{\theta}=\operatorname{ker} d_{0} \Phi_{\theta}=\left\{\nu \in L^{\infty}\left(\Gamma_{\theta}\right): \iint_{U / \Gamma_{\theta}} \nu \psi=0, \text { for all } \psi \in Q\left(\Gamma_{\theta}\right)\right\} .
$$

But there is a Banach manifold isomorphism $\omega_{\theta}^{*}: M\left(\Gamma_{\theta}\right) \rightarrow M(\Gamma)$ defined by: $\omega_{\theta}^{*}(\nu)=$ complex dilatation of $\left(w_{\nu} \circ w_{\theta}\right)$. Indeed a calculation shows that $\omega_{\theta}^{*}$ is biholomorphic and it induces a $C^{1}$-diffeomorphism $\xi_{\theta}: T\left(\Gamma_{\theta}\right) \rightarrow$ $T(\Gamma)$ so that

$$
\xi_{\theta} \circ \Phi_{\theta}=\Phi \circ \omega_{\theta}^{*}: M\left(\Gamma_{\theta}\right) \rightarrow T(\Gamma) .
$$

Hence,

$$
d_{\Phi_{\theta}(0)} \xi_{\theta} \circ d_{0} \Phi_{\theta}=d_{\theta} \Phi \circ d_{0} \omega_{\theta}^{*}
$$

and so

$$
\operatorname{ker} d_{\theta} \Phi=d_{0} \omega_{\theta}^{*}\left(\operatorname{ker} d_{0} \Phi_{\theta}\right)=d_{0} \omega_{\theta}^{*}\left(K_{\theta}\right) .
$$

Now when $\theta$ is in the fiber of $\Phi$ above $\Phi(0)$ we immediately notice that $\Gamma_{\theta} \equiv \Gamma$ and then $K_{\theta}$ is the same complex subspace as $K_{0}$.

So ker $d_{\theta} \Phi=\left(d_{0} \omega_{\theta}^{*}\right)\left(K_{0}\right)\left(\subset L^{\infty}(\Gamma)\right)$. Since $\omega_{\theta}^{*}$ was holomorphic and $K_{0}$ is a complex subspace we see that ker $d_{\theta} \Phi$ is also a complex subspace of $L^{\infty}(\Gamma)$ which varies continuously with $\theta$ in the fiber (because $d_{0} \omega_{\theta}^{*}$ varies continuously with $\theta)$. Choose a fixed $(3 g-3+k)$-dimensional subspace $G_{0}$ complementary to $K_{0}$ in $L^{\infty}(\Gamma)$. Then, since ker $d_{\theta} \Phi$ varies continuously, for some neighbourhood of values of $\theta$ (around 0 ) in the 
fiber, $G_{0}$ must remain complementary to each of the complex subspaces $\operatorname{ker} d_{\theta} \Phi$ identified above. Hence, over this neighbourhood in the fiber the map $\Phi$ induces a well-defined complex structure to the tangent space to $T(\Gamma)$ at $\Phi(0)$, viz. the complex structure of $G_{0}$.

Now, if two neighbourhoods as above on the fiber overlap then $\Phi$ induces the same complex-structure on the tangent space to $T(\Gamma)$ at $\Phi(0)$ over the full union of the two neighbourhoods since any point in the intersection of the two determines this complex structure.

But we claim that the fibers of $\Phi$ are path connected. For if $\Phi(\mu)=$ $\Phi(\nu)$ then the maps $w_{\mu}$ and $w_{\nu}$ induce homotopic maps $f_{\mu}$ and $f_{\nu}$ from $U / \Gamma$ to $U / \Gamma_{\mu}$. We choose a homotopy between $f_{\mu}$ and $f_{\nu}$ via quasiconformal maps and thus obtain a path of Beltrami differentials joining $\mu$ and $\nu$ and lying in the same fiber of $\Phi$.

An obvious compactness argument following from the path-connectedness of the fibers now shows that $\Phi$ induced a well defined almost complex structure on $T(\Gamma)$. Applying Theorem 3 we therefore obtain the existence of the natural complex structure on $T(\Gamma)$ making $\Phi$ a holomorphic submersion.

REMARK. This application was the original motivation for the general problem tackled in this paper. The author thanks B. A. Taylor for some helpful discussions regarding Theorem 3 and its application.

$A$ counterexample. It may be thought that in the situation we are studying if $f: X \rightarrow Y$ has its fibers as complex manifolds of the correct dimension in $X$, then already this guarantees that $Y$ has a complex structure making $f$ holomorphic. That this is not the case is demonstrated by the following general counterexample, which serves to establish the irredundancy of the conditions of Theorems 2 and 3.

Let $M$ be a compact oriented $C^{\infty}$-surface of finite type. Consider the Teichmuller space $T(M)$ of Riemann Surfaces modelled on $M$ and the universal curve $V(M) \stackrel{p}{\rightarrow} T(M)$ (for relevant definitions see Earle [2]). The fiber of the projection $p$ above a point $t \in T(M)$ is the Riemann surface represented by $t$. From Teichmuller theory we know that any neighborhood of a point in $T(M)$ has points representing holomorphically distinct Riemann surfaces. Thus any restriction of the universal curve

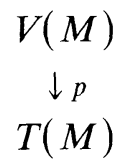


to an open subset $S$ of $T(M)$,

$$
\begin{aligned}
& \left.V(M)\right|_{S}=p^{-1}(S) \\
& p \downarrow \\
& S \subset T(M)
\end{aligned},
$$

gives a 'marked family of Riemann surfaces' which is not analytically the product of $S$ with any fixed Riemann surface. Now we have the following result (Kuranishi [4], Chapter IV).

Proposition 1. There is a diffeomorphism

$$
\begin{array}{ccc}
\gamma: V(M) & \rightarrow & T(M) \times M \\
p \searrow & & \swarrow \pi_{1} \\
& T(M) &
\end{array}
$$

such that the diagram commutes. We will call $\gamma$ a 'marking-map' for $V(M)$. There exists a neighbourhood $S$ of any point of $T(M)$ and a marking-map $g$ such that $\left.V(M)\right|_{s}$ has 'horizontally analytic sections', viz.

$$
\begin{array}{ccc}
\left.V(M)\right|_{S} & \stackrel{g}{\rightarrow} & S \times M \\
p \searrow & & \swarrow \pi_{1} \\
& S &
\end{array}
$$

and $\left.g^{-1}\right|_{S \times\{m\}}: S \times\left.\{m\} \rightarrow V(M)\right|_{S}$ is analytic for all $m \in M$.

THEOREM 5. Let $S$ be an open subset of $T(M)$ which allows a markingmap $g$ with horizontally analytic sections as above. Let $X=\left.V(M)\right|_{s}, X$ is a complex manifold of dimension $m$, say. Then the map $f: X \rightarrow M$ given by $f=\pi_{2} \circ g$ is a surjective submersion and has fibers which are complex submanifolds of $X$ of dimension $(m-1)$; but $M$ has no complex structure on it making $f$ holomorphic.

Proof.

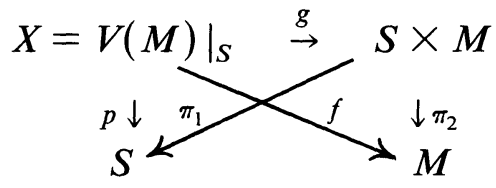

That $f$ is a surjective submersion is clear.

The fiber of $f$ above $m \in M$ is simply the image of $\left.g^{-1}\right|_{S \times\{m\}}$, which is a complex submanifold of $X$ isomorphic to $S$ (because $g$ had horizontally analytic sections). 
Suppose there is a complex structure $\sigma$ on $M$ such that $f: X \rightarrow M$ is holomorphic. Then $\pi_{1} \circ g=p$ and $\pi_{2} \circ g=f$ are now holomorphic. So $g$ is holomorphic, exhibiting $X$ as an analytic product of $S$ with a fixed Riemann surface $M$. This is a contradiction.

An explicit case of the above type is furnished by an analytic family of tori over the upper half-plane $U$. Let $X=(U \times \mathbf{C}) / z \times Z$ where $Z \times Z$ acts discontinuously on $U \times \mathbf{C}$ by identifying $(\tau, z)$ with $(\tau, z+m+n \tau)$, for all $(m, n) \in Z \times Z$. Let $M=\mathrm{C} /$ lattice generated by $(1$ and $i)=\mathrm{a}$ topological torus. Define $f: X \rightarrow M$ by

$$
(\tau,[a+b \tau]) \stackrel{f}{\rightarrow}[a+b i], \quad a, b \in \mathbf{R},
$$

([ $\cdot]$ denotes the appropriate equivalence class of the point). The fibers of this submersive $f$ are all complex submanifolds in $X$, each isomorphic to $U$. But if there were a complex structure $\sigma$ on $M$ making $f$ holomorphic, then $U \times \mathbf{C} / Z \times Z$ would be analytically the product of $U$ and $M_{\sigma}$, which it is not.

The author is grateful to J. Hubbard for suggesting the idea behind the above 'counterexample'.

Some applications of the above results will appear in a forthcoming paper of the author entitled "On some Involutions and Retractions arising in Teichmuller Spaces", in The Journal of the Indian Mathematical Society.

\section{REFERENCES}

[1] A. Dold, Lectures on Algebraic Topology, Springer-Verlag, Berlin, New York, 1972.

[2] C. J. Earle, Teichmuller theory, in Discrete Groups and Automorphic Functions, (Harvey, ed.), Academic Press, 1977.

[3] M. Herve, Several Complex Variables, Local Theory, Oxford University Press, London, New York, 1963 (published for TIFR, Bombay).

[4] M. Kuranishi, Deformations of Compact Complex Manifolds, Lecture notes, Univ. of Montreal, Summer 1969.

[5] F. Warner, Foundations of Differentiable Manifolds and Lie Groups, Scott Foresman, Glenview, Illinois, 1971.

Received February 16, 1982. This research is based partly on the author's $1980 \mathrm{Ph} . \mathrm{D}$. thesis at Cornell University. The author thanks his thesis adivsor, Professor C. J. Earle, for his support and help. I thank the referees for correcting an oversight and helpful comments.

The author is currently a Visiting Fellow in the Tata Institute of Fundamental Research.

TATA INSTITUTE OF FUNDAMENTAL RESEARCH

HOMI BHABHA ROAD

BOMBAY 400 005, INDIA 



\section{PACIFIC JOURNAL OF MATHEMATICS \\ EDITORS}

DONALD BABBITT (Managing Editor)

University of California

Los Angeles, CA 90024

Hugo RossI

University of Utah

Salt Lake City, UT 84112

C. C. MOOre and ARThur OGus

University of California

Berkeley, CA 94720
J. Dugundi

Department of Mathematics

University of Southern California

Los Angeles, CA 90089-1113

R. FINN and H. SAMELSON

Stanford University

Stanford, CA 94305

ASSOCIATE EDITORS

$\begin{array}{lllll}\text { R. Arens } & \text { E. F. Beckenbach } & \text { B. H. Neumann } & \text { F. Wolf } & \text { K. Yoshida }\end{array}$ $(1906-1982)$

\section{SUPPORTING INSTITUTIONS}

UNIVERSITY OF ARIZONA

UNIVERSITY OF BRITISH COLUMBIA

CALIFORNIA INSTITUTE OF TECHNOLOGY

UNIVERSITY OF CALIFORNIA

MONTANA STATE UNIVERSITY

UNIVERSITY OF NEVADA, RENO

NEW MEXICO STATE UNIVERSITY

OREGON STATE UNIVERSITY
UNIVERSITY OF OREGON

UNIVERSITY OF SOUTHERN CALIFORNIA

STANFORD UNIVERSITY

UNIVERSITY OF HAWAII

UNIVERSITY OF TOKYO

UNIVERSITY OF UTAH

WASHINGTON STATE UNIVERSITY

UNIVERSITY OF WASHINGTON 


\section{Pacific Journal of Mathematics}

\section{Vol. 110, No. $1 \quad$ September, 1984}

Wojciech Abramczuk, A class of surjective convolution operators $\ldots \ldots \ldots \ldots 1$

K. Adachi, Extending bounded holomorphic functions from certain

subvarieties of a weakly pseudoconvex domain $\ldots \ldots \ldots \ldots \ldots \ldots$

Malvina Florica Baica, An algorithm in a complex field and its application

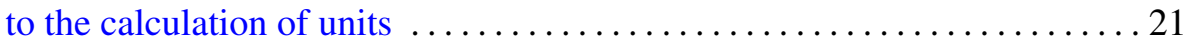

Giuliana Bianchi and Robert Cori, Colorings of hypermaps and a

conjecture of Brenner and Lyndon $\ldots \ldots \ldots \ldots \ldots \ldots \ldots \ldots \ldots \ldots \ldots \ldots$

Ronald James Evans, Determinations of Jacobsthal sums . . .......... 49

Leslie Foged, Characterizations of $\aleph$-spaces .................... 59

Nassif A. Ghoussoub and Paulette Saab, Weak compactness in spaces of

Bochner integrable functions and the Radon-Nikodým property . . . . . . 65

J. Gómez Gil, On local convexity of bounded weak topologies on Banach

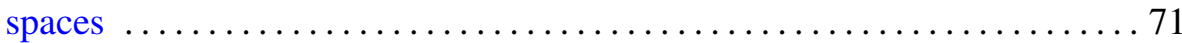

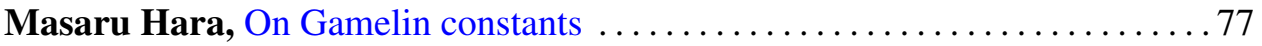

Wilfried Hauenschild, Eberhard Kaniuth and Ajay Kumar, Harmonic

analysis on central hypergroups and induced representations $\ldots \ldots \ldots 83$

Eugenio Hernandez, An interpolation theorem for analytic families of

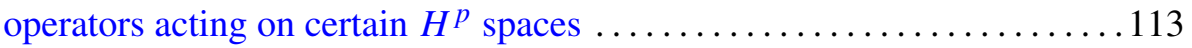

Thomas Alan Keagy, On "Tauberian theorems via block-dominated

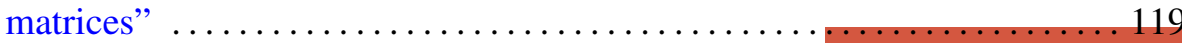

Thomas Landes, Permanence properties of normal structure $\ldots \ldots \ldots \ldots \ldots 125$

Daniel Henry Luecking, Closed ranged restriction operators on weighted

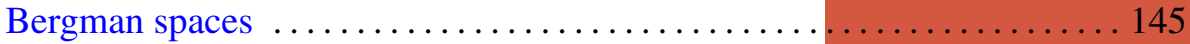

Albert Thomas Lundell, The $p$-equivalence of $\mathrm{SO}(2 n+1)$ and $\mathrm{Sp}(n) \ldots \ldots 161$

Mark D. Meyerson, Remarks on Fenn's "the table theorem" and Zaks' "the chair theorem" ..................................... 167

Marvin Victor Mielke, Homotopically trivial toposes . . . . . . . . . . 171

Gerard J. Murphy, Hyperinvariant subspaces and the topology on Lat A . . 183

Subhashis Nag, On the holomorphy of maps from a complex to a real manifold

Edgar Milan Palmer and Robert William Robinson, Enumeration of self-dual configurations ................................. 203

John J. Walsh and David Clifford Wilson, Continuous decompositions

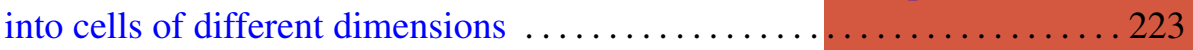

Walter John Whiteley, Infinitesimal motions of a bipartite framework .....233 